\title{
Differences Between Dietary, Drinking Habits and Physical Activity of the Adult Population Living in Two Distinct Geographic Areas of Romania
}

\author{
IOSIF BALINT, SANDOR PAL*, LORAND FERENCZ, ZOLTAN ABRAM \\ UMPhST Tirgu Mures, Faculty of Medicine, 38 Gh. Marinescu, 540139, Tirgu Mures, Romania
}

\begin{abstract}
The constantly and intensely changing modern society has a negative influence on the the general population's health status. Risk factors being deeply inserted in the structure of the modern society can hardly be influenced and corrected. The aim of the study was the lifestyle- and diet-related comparison of two distinct adult populations from Romania: Bacau and Tirgu Mures. Material and methods: We evaluated the dietary habits, alcohol consumption and physical activity by a complex, validated questionnaire. Body mass index was also determined. The adultpopulation from Tirgu Mures numbered 352 subjects, who were compared to the adults from Bacau, numbering 231 individuals. The considered threshold of significance was 0.05 . Body mass index is over the normal range in more than 2/3 of the subjects. The overweight occurrence was $37.95 \%$. The majority of the subjects (77.5\%) has regular daily exercise (30 minutes or more). Daily alcohol consumption was present in $42.42 \%$ of the cases. Dietary habits were also analyzed: $61.9 \%$ of the subjects consume breakfast daily, and $46.94 \%$ of them consumes snacks between meals $2-3$ times per week. $A$ statistical difference could be observed regarding the frequency of breakfast consumption and body mass index (p-0.042): $2 / 3$ of the studied adults from Tirgu Mures who eat breakfast sometimes having their body mass index over $25 \mathrm{~kg} / \mathrm{m}^{2}$. Regarding physical activity a significant difference could be observed in case of those who responded rarely or never ( $p-0.027)$ (76.0\% of Mures county with this response vs. only $24.0 \%$ in Bacau). The frequency of alcohol consumption is higher in Tirgu Mures and surroundings in case of once or several times per week consumption ( $p$-0.005). Instructional and educational interventions targeting the development of healthier lifestyle habits in general population are worthy and needed to reduce the risk factors associated with the development of cardiovascular and metabolic diseases and reducing the burden upon the society of these diseases.
\end{abstract}

Keywords: alcohol consumption, dietary habits, lifestyle, state of health

The modern society is characterized by constant and intense changes, having often negative influence on the state of health. Several modern lifestyle habits increase the risk for developing diseases, including obesity [1]. Sometimes the risk factors become so deeply inserted in the structure of the modern society that influencing them is almost impossible [2]. Spaces for socialization have an important role in the development of a sedentary lifestyle, lack of physical exercise having an important negative impact on maintaining the state of health.

The state of health is also affected by other social factors like globalization, social status, education and poor life conditions. Thus, several problems related to the state of health are strongly associated to lifestyle habits, such as alcohol consumption, smoking and inadequate diet [3]. Many of these factors increase the level of oxidative stress, a highly studied process in which the amount of reactive oxygen species exceeds the neutralization capacity of the body, damaging the biomolecules and inducing diseases [4].

Sociological studies identified certain unfavored social groups being vulnerable to different diseases or to have habits representing risk on the state of health. Social factors can become protective on other population groups by social services or prevention/education programs. The variety of the impact exhibited by these factors explains the differences between certain social environments regarding the occurrence of diseases and the state of health [5].
Natural and social environment, available financial and psychological resources, problem solving capacity, success and satisfaction altogether can become factors that contribute to the state of health. Thus, health can be defined as a continuous process depending on multiple factors. State of health can be considered a dynamic equilibrium in the relationship of the individual with the social environment, which permits increased life expectancy and better quality of life, and losing it represents a social problem influencing the entire society [6].

Healthy habits are the result of natural tendencies of people, while several habits representing risk for the state of health (such as smoking and alcohol consumption) are the result of individual decisions. These decisions influence the state of health and have an important impact on the lifestyle [7]. The recommended healthylifestyle habits are to drink water of a low mineral content $0.03 x$ bodyweight, to do regular and intensive exercise 30 minutes daily due to blood circulation and to eat a fibre rich diet, because the cellulose fibres from fruits and vegetables have negatively charged hydrophilic surfaces along which a compact water structure can be formed. Fiber-diet is essential especially in prevention of cancer and degenerative diseases, being based on vegetable, fruit and whole cereal intake $[8,9]$. Another major problem nowadays is represented by the high incidence of autoimmune diseases; some of them are diet-related, like celiac disease [10].

Nowadays public health programs are more and more focused on diversity and on the special needs of certain 
vulnerable groups. Obesity is one of the problems strictly related to cultural and social factors [11, 12]. Weight presents a seasonal variation, depending on the intensity of physical activity and related to the amount of food intake [13]. According to a Romanian report, obesity and overweight could be prevented with a proper diet, which is also cost-effective and simple to be introduced to a wide range of population [14].

The aim of the study was to compare lifestyle and dietary habits of adult population living in two distinct geographic areas of Romania: Bacau and surroundings versus Tirgu Mures and surroundings.

\section{Experimental part}

Evaluation of dietary habits, drink consumption and physical activity was performed by a complex validated questionnaire. Body mass index (BMI) was determined based on the height and weight of the patients. The adult population from Tirgu Mures and surroundings numbered 352 subjects, their results were compared to the adults from Bacau and surroundings ( 231 individuals). The study population was aged between 19-64 years.

Chi-square and t-Student tests were applied for statistics, SPSS and GraphPad InStat programs being used for this purpose. Statistical significance was determined based on $p$ value, the considered threshold for significance was 0.05 .

\section{Results and discussions}

Body mass index (BMI) of the studied population was calculated, and according to our results we can state that in more than two third of the subjects, BMI is over the normal range (68.31\%). Overweight was most frequent in the studied population (37.95\%) (fig. 1).

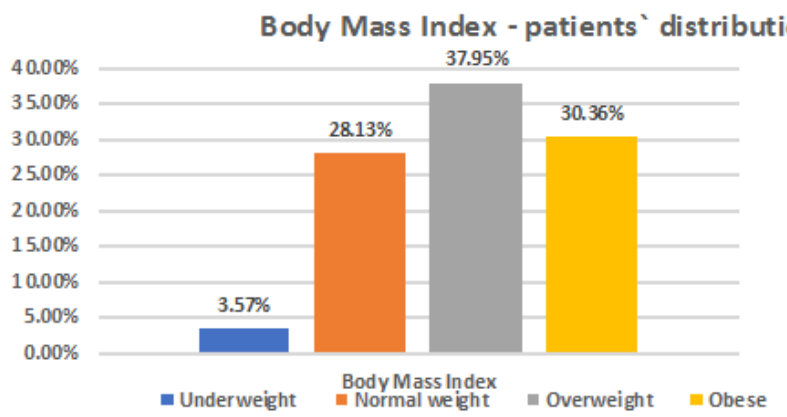

Fig. 1. Distribution of population according to their BMI

Based on the questions regarding physical activity, we can state that the majority of the subjects (77.5\%) have regular exercise daily or at least during working days ( 30 min or more). In only $2.6 \%$ of the respondents we could observe the lack of this activity (fig. 2).

Evaluating the frequency of alcohol consumption we can observe a high percentage of patients with daily alcohol consumption (42.42\%), from these $18.61 \%$ consumes more than one portion per day, and $46.32 \%$ weekly (fig. 3).

Regarding eating habits, breakfast is consumed daily by more than half of the studied subjects (61.9\%) (table 1). Snacks between the main meals are less frequent: the highest percentage (46.94\%) consumes snacks 2-3 times per week (fig. 4).

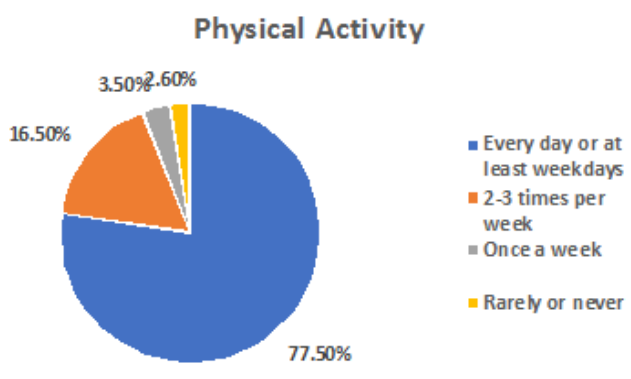

Fig. 2. Distribution of population regarding physical activity Alcohol consumption

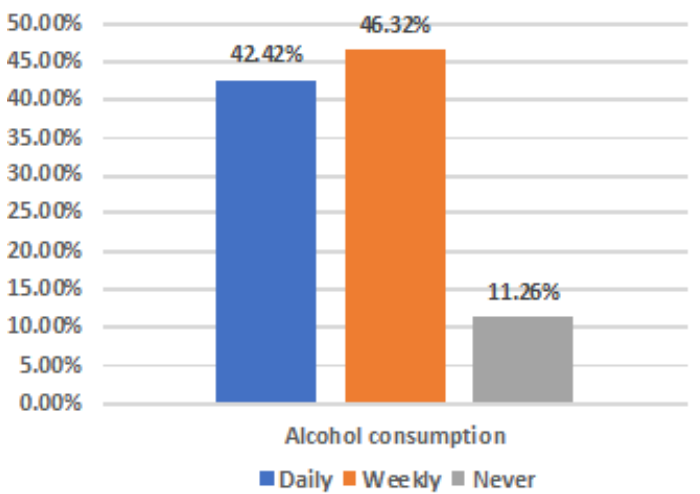

Fig. 3. Percentage of responses regarding alcohol consumption Snacks

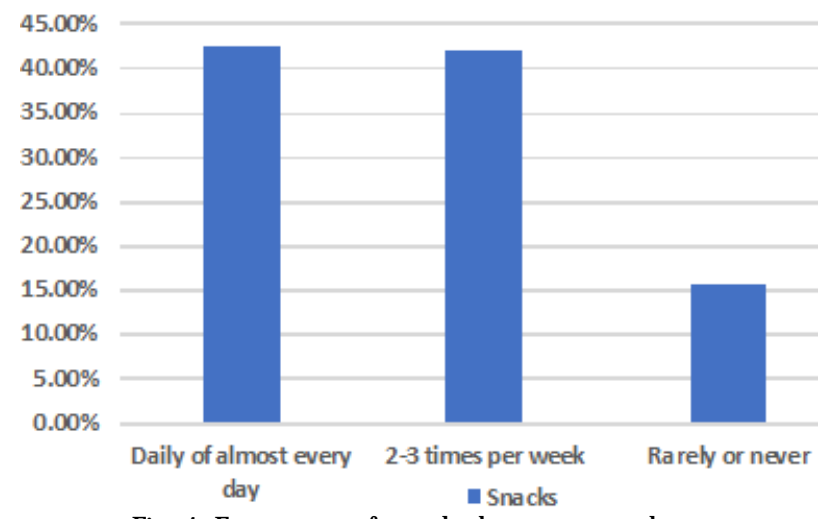

Fig. 4. Frequency of snacks between meals

In order to reveal statistical differences between the population living in the two distinct geographic areas (Bacau and Mures) regarding lifestyle habits (food intake, physical excercise, alcohol consumption), we applied the chi square test. We combined the BMl classes with the parameters of the populations from these two areas.

We studied the relationship between BMI and breakfast eating habits (table 2). No significant associations could be observed with $\mathrm{BMI}$ values in the two studied populations from Bacau ( $p-0.66)$ or Tirgu Mures and surroundings ( $p$ 0.59 ). We observed that more than half of the adults having normal BMI or being overweight eat breakfast daily. No statistical significant differences could be revealed for eating breakfast daily or almost never ( $p-0.09)$, and a similar outcome was obtained comparing those that eat breakfast rarely or never ( $p-0.44)$. Significant difference occured for eating breakfast sometimes ( $p-0.042), 2 / 3$ of the studied adults in Tirgu Mures and surroundings having BMI over $25 \mathrm{~kg} / \mathrm{cm}^{2}$ eat breakfast sometimes (table 2).

\begin{tabular}{|l|l|l|l|l|l|l|}
\hline Breakfast & \multicolumn{9}{|l|}{ Mures } & \multicolumn{1}{l|}{ Total } \\
\hline & Bacau & \multicolumn{1}{|c|}{} & \\
\hline Daily & 127 & $55.0 \%$ & 234 & $66.5 \%$ & 361 & $61.9 \%$ \\
\hline Sometimes & 53 & $22.9 \%$ & 104 & $29.5 \%$ & 157 & $26.9 \%$ \\
\hline Rarely/never & 51 & $22.1 \%$ & 14 & $4 \%$ & 65 & $11.1 \%$ \\
\hline Total & 231 & $100.0 \%$ & 352 & $100.0 \%$ & 583 & $100.0 \%$ \\
\hline
\end{tabular}

Table 1

NUMBER AND PERCENTAGE OF SUBJ ECT REGARDING THE FREQENCY OF BREAKFAST CONSUPTION 


\begin{tabular}{|c|c|c|c|c|c|c|c|c|c|}
\hline & Breakfa & & & & & BMI & & & Total \\
\hline Daily or & Groups & Bacau & $\mathrm{nr}$. & 4 & 29 & 56 & 26 & 12 & 127 \\
\hline almost & & & $\%$ & $66.7 \%$ & $35.4 \%$ & $32.0 \%$ & $33.8 \%$ & $57.1 \%$ & $35.2 \%$ \\
\hline daily p- & & Mures & nr. & 2 & 53 & 119 & 51 & 9 & 234 \\
\hline & & & $\%$ & $33.3 \%$ & $64.6 \%$ & $68.0 \%$ & $66.2 \%$ & $42.9 \%$ & $64.8 \%$ \\
\hline & Total & & nr. & 6 & 82 & 175 & 77 & 21 & 361 \\
\hline & & & $\%$ & 100.0 & 100.0 & 100.0 & 100.0 & 100.0 & 100.0 \\
\hline Some- & Groups & Bacau & $\mathrm{nr}$. & 2 & 19 & 16 & 12 & 4 & 53 \\
\hline $\begin{array}{l}\text { times } \mathrm{p}- \\
0.042\end{array}$ & & & $\%$ & $\begin{array}{l}100.0 \\
\%\end{array}$ & $46.3 \%$ & $24.2 \%$ & $33.3 \%$ & $33.3 \%$ & $33.8 \%$ \\
\hline & & Mures & nr. & 0 & 22 & 50 & 24 & 8 & 104 \\
\hline & & & $\%$ & $0.0 \%$ & $53.7 \%$ & $75.8 \%$ & $66.7 \%$ & $66.7 \%$ & $66.2 \%$ \\
\hline & Total & & $\mathrm{nr}$. & 2 & 41 & 66 & 36 & 12 & 157 \\
\hline & & & $\%$ & 100.0 & 100.0 & $\begin{array}{l}100.0 \\
\%\end{array}$ & $\begin{array}{l}100.0 \\
\%\end{array}$ & 100.0 & 100.0 \\
\hline Rarely or & Groups & Bacau & nr. & 2 & 10 & 21 & 12 & 6 & 51 \\
\hline $\begin{array}{l}\text { almost } \\
\text { never }\end{array}$ & & & $\%$ & $\begin{array}{l}100.0 \\
\%\end{array}$ & $66.7 \%$ & $75.0 \%$ & $92.3 \%$ & $85.7 \%$ & $78.5 \%$ \\
\hline p-0.44 & & Mures & $\mathrm{nr}$. & 0 & 5 & 7 & 1 & 1 & 14 \\
\hline & & & $\%$ & $0.0 \%$ & $33.3 \%$ & $25.0 \%$ & $7.7 \%$ & $14.3 \%$ & $21.5 \%$ \\
\hline & Total & & $\mathrm{nr}$. & 2 & 15 & 28 & 13 & 7 & 65 \\
\hline & & & $\%$ & 100.0 & 100.0 & 100.0 & 100.0 & 100.0 & 100.0 \\
\hline Total & Groups & Bacau & nr. & 8 & 58 & 93 & 50 & 22 & 231 \\
\hline $\mathrm{p}-0.005$ & & & $\%$ & $80.0 \%$ & $42.0 \%$ & $34.6 \%$ & $39.7 \%$ & $55.0 \%$ & $39.6 \%$ \\
\hline & & Mures & $\mathrm{nr}$. & 2 & 80 & 176 & 76 & 18 & 352 \\
\hline & & & $\%$ & $20.0 \%$ & $58.0 \%$ & $65.4 \%$ & $60.3 \%$ & $45.0 \%$ & $60.4 \%$ \\
\hline & Total & & $\mathrm{nr}$. & 10 & 138 & 269 & 126 & 40 & 583 \\
\hline & & & $\%$ & $\begin{array}{l}100.0 \\
\%\end{array}$ & $\begin{array}{l}100.0 \\
\%\end{array}$ & $\begin{array}{l}100.0 \\
\%\end{array}$ & $\begin{array}{l}100.0 \\
\%\end{array}$ & $\begin{array}{l}100.0 \\
\%\end{array}$ & $\begin{array}{l}100.0 \\
\%\end{array}$ \\
\hline
\end{tabular}

Table 2

RELATIONSHIP OF BMI AND BREAKFAST EATING HABITS OF THE TWO STUDIED GROUPS
Regarding snacks between the main meals, their consumption is different between the two geographic regions in the perspective of frequency. In Bacau the responders consume snacks frequently, sometimes and rarely or never, without significant association with BMI values ( $p-0.57)$. In case of the adults in Tirgu Mures and surroundings, the frequencies were daily, almost daily, 2-3 times per week and rarely or never, but without significant association with BMI ( $p-0.17)$.
Statistical significant differences could be revealed only in the situation of consuming snacks rarely or never, which option was present in case of both populations, $p$ value being 0.044 . The difference occurred in case of the group having BMI under $18.5 \mathrm{~kg} / \mathrm{cm}^{2}$ (table 3 ).

Comparing the results obtained in the two areas regarding physical activity revealed differences only in case of having physical excercise rarely or never, where $p$ value was 0.027 . For the other frequencies of physical activity we could

\begin{tabular}{|c|c|c|c|c|c|c|c|c|}
\hline \multirow{2}{*}{\multicolumn{3}{|c|}{ Suacks }} & \multicolumn{5}{|c|}{ BMII } & \multirow[t]{2}{*}{ Total } \\
\hline & & & 218.5 & $18.5-24.9$ & $25.0-29.9$ & $30.0-34.9$ & $35.0-39.9$ & \\
\hline \multicolumn{9}{|c|}{ Daily or almost daily (p-could not be calculated) } \\
\hline \multirow{4}{*}{ Group } & \multirow[t]{2}{*}{ Mures } & nir & 1 & 18 & 28 & 6 & 4 & 57 \\
\hline & & $\%$ & $100.0 \%$ & $100.0 \%$ & $100.0 \%$ & $100.0 \%$ & $100.0 \%$ & $100.0 \%$ \\
\hline & \multirow[t]{2}{*}{ Total } & $\mathrm{nr}$ & 1 & 18 & 28 & 6 & 4 & 57 \\
\hline & & $\%$ & $100.0 \%$ & $100.0 \%$ & $100.0 \%$ & $100.0 \%$ & $100.0 \%$ & $100.0 \%$ \\
\hline \multicolumn{9}{|c|}{$2-3$ times per weel ( $\mathrm{p}$ - could not be calculated) } \\
\hline \multirow[t]{4}{*}{ Group } & \multirow[t]{2}{*}{ Mures } & $\mathrm{nr}$ & 1 & 19 & 58 & 24 & 7 & 109 \\
\hline & & $\%$ & $100.0 \%$ & $100.0 \%$ & $100.0 \%$ & $100.0 \%$ & $100.0 \%$ & $100.0 \%$ \\
\hline & \multirow[t]{2}{*}{ Total } & $\mathrm{nr}$ & 1 & 19 & 58 & 24 & 7 & 109 \\
\hline & & $\%$ & $100.0 \%$ & $100.0 \%$ & $100.0 \%$ & $100.0 \%$ & $100.0 \%$ & $100.0 \%$ \\
\hline \multicolumn{9}{|c|}{ Frequent (p-could not be calculated) } \\
\hline \multirow[t]{4}{*}{ Group } & \multirow[t]{2}{*}{ Bacau } & $\mathrm{nr}$ & 3 & 11 & 13 & 6 & 3 & 36 \\
\hline & & $\%$ & $100.0 \%$ & $100.0 \%$ & $100.0 \%$ & $100.0 \%$ & $100.0 \%$ & $100.0 \%$ \\
\hline & \multirow[t]{2}{*}{ Total } & nI & 3 & 11 & 13 & 6 & 3 & 36 \\
\hline & & $\%$ & $100.0 \%$ & $100.0 \%$ & $100.0 \%$ & $100.0 \%$ & $100.0 \%$ & $100.0 \%$ \\
\hline \multicolumn{9}{|c|}{ Sometimes ( $\mathrm{p}$ - could not be calculated) } \\
\hline \multirow[t]{4}{*}{ Group } & \multirow[t]{2}{*}{ Bacau } & nI & 2 & 27 & 35 & 23 & 10 & 97 \\
\hline & & $\%$ & $100.0 \%$ & $100.0 \%$ & $100.0 \%$ & $100.0 \%$ & $100.0 \%$ & $100.0 \%$ \\
\hline & \multirow[t]{2}{*}{ Total } & nI & 2 & 27 & 35 & 23 & 10 & 97 \\
\hline & & $\%$ & $100.0 \%$ & $100.0 \%$ & $100.0 \%$ & $100.0 \%$ & $100.0 \%$ & $100.0 \%$ \\
\hline \multicolumn{9}{|c|}{ Rarely or never p-0.044 } \\
\hline \multirow[t]{6}{*}{ Group } & \multirow[t]{2}{*}{ Bacau } & $\mathrm{nr}$ & 3 & 20 & 45 & 21 & 9 & 98 \\
\hline & & $\%$ & $100.0 \%$ & $31.7 \%$ & $33.3 \%$ & $31.3 \%$ & $56.2 \%$ & $34.5 \%$ \\
\hline & \multirow[t]{2}{*}{ Mures } & $\mathrm{nr}$ & 0 & 43 & 90 & 46 & 7 & 186 \\
\hline & & $\%$ & $0.0 \%$ & $68.3 \%$ & $66.7 \%$ & $68.7 \%$ & $43.8 \%$ & $65.5 \%$ \\
\hline & \multirow[t]{2}{*}{ Total } & $\mathrm{nr}$ & 3 & 63 & 135 & 67 & 16 & 284 \\
\hline & & $\%$ & $100.0 \%$ & $100.0 \%$ & $100.0 \%$ & $100.0 \%$ & $100.0 \%$ & $100.0 \%$ \\
\hline \multicolumn{9}{|c|}{ Total p-008 } \\
\hline \multirow[t]{6}{*}{ Group } & \multirow[t]{2}{*}{ Bacau } & $\mathrm{nI}$ & 8 & 58 & 93 & 50 & 22 & 231 \\
\hline & & $\%$ & $80.0 \%$ & $42.0 \%$ & $34.6 \%$ & $39.7 \%$ & $55.0 \%$ & $39.6 \%$ \\
\hline & Mures & $\mathrm{nr}$ & 2 & 80 & 176 & 76 & 18 & 352 \\
\hline & & $\%$ & $20.0 \%$ & $58.0 \%$ & $65.4 \%$ & $60.3 \%$ & $45.0 \%$ & $60.4 \%$ \\
\hline & Total & $\mathrm{nr}$ & 10 & 138 & 269 & 126 & 40 & 583 \\
\hline & & $\%$ & $100.0 \%$ & $100.0 \%$ & $100.0 \%$ & $100.0 \%$ & $100.0 \%$ & $100.0 \%$ \\
\hline
\end{tabular}

COMPARATIVE RESPONSE RATES IN THE STUDIED GROUPS REGARDING THE FREQUENCY OF SNACKS BETWEEN MEALS 
observe that the adults living in Tirgu Mures and

surroundings have more frequently activities involving

physical effort compared to the adults in Bacau, but the differences are not statistically significant (table 4).

\begin{tabular}{|c|c|c|c|c|c|c|c|c|}
\hline \multirow{2}{*}{\multicolumn{3}{|c|}{ Alcohol(vine, beer, liqueur) }} & \multicolumn{5}{|c|}{ BMI } & \multirow[t]{2}{*}{ Total } \\
\hline & & & $<18.5$ & $18.5-24.9$ & $25.0-29.9$ & $30.0-34.9$ & $35.0-39.9$ & \\
\hline \multicolumn{9}{|c|}{ Adaily portion $\mathrm{p}-0.45$} \\
\hline \multirow[t]{6}{*}{ Groups } & \multirow[t]{2}{*}{ Bacalu } & $\mathrm{nr}$ & 1 & 16 & 19 & 17 & 2 & 55 \\
\hline & & $\%$ & $50.0 \%$ & $66.7 \%$ & $61.3 \%$ & $85.0 \%$ & $66.7 \%$ & $68.8 \%$ \\
\hline & \multirow[t]{2}{*}{ Mures } & $\mathrm{nr}$ & 1 & 8 & 12 & 3 & 1 & 25 \\
\hline & & $\%$ & $50.0 \%$ & $33.3 \%$ & $38.7 \%$ & $15.0 \%$ & $33.3 \%$ & $31.2 \%$ \\
\hline & \multirow[t]{2}{*}{ Total } & $\mathrm{nr}$ & 2 & 24 & 31 & 20 & & \\
\hline & & $\%$ & $100.0 \%$ & $100.0 \%$ & $100.0 \%$ & $100.0 \%$ & $100.0 \%$ & $100.0 \%$ \\
\hline \multicolumn{9}{|c|}{ Maltiple daily portious $\mathrm{p}-0.7 \mathrm{t}$} \\
\hline \multirow{6}{*}{ Groups } & \multirow[t]{2}{*}{ Bacall } & $\mathrm{nr}$ & 2 & 11 & 18 & 4 & 8 & 43 \\
\hline & & $\%$ & $100.0 \%$ & $91.7 \%$ & $85.7 \%$ & $80.0 \%$ & $100.0 \%$ & $89.6 \%$ \\
\hline & \multirow[t]{2}{*}{ Nures } & $\mathrm{nr}$ & 0 & 1 & 3 & 1 & 0 & 5 \\
\hline & & $\%$ & $0.0 \%$ & $8.3 \%$ & $14.3 \%$ & $20.0 \%$ & $0.0 \%$ & $10.4 \%$ \\
\hline & \multirow[t]{2}{*}{ Total } & $\mathrm{nr}$ & 2 & 12 & 21 & 5 & 8 & \\
\hline & & $\%$ & $100.0 \%$ & $100.0 \%$ & $100.0 \%$ & $100.0 \%$ & $100.0 \%$ & $100.0 \%$ \\
\hline \multicolumn{9}{|c|}{ A weellily portion or less $\mathrm{p}-0.005$} \\
\hline \multirow[t]{6}{*}{ Groups } & \multirow[t]{2}{*}{ Bacall } & nI & 1 & 11 & 18 & 5 & 6 & 41 \\
\hline & & $\%$ & $100.0 \%$ & $26.2 \%$ & $15.7 \%$ & $10.9 \%$ & $46.2 \%$ & $18.9 \%$ \\
\hline & \multirow[t]{2}{*}{ Mures } & $\mathrm{nr}$ & 0 & 31 & 97 & 41 & & 176 \\
\hline & & $\%$ & $0.0 \%$ & $73.8 \%$ & $84.3 \%$ & $89.1 \%$ & $53.8 \%$ & $81.1 \%$ \\
\hline & \multirow[t]{2}{*}{ Total } & ar & 1 & 42 & 115 & 46 & 13 & 217 \\
\hline & & $\%$ & $100.0 \%$ & $100.0 \%$ & $100.0 \%$ & $100.0 \%$ & $100.0 \%$ & $100.0 \%$ \\
\hline \multicolumn{9}{|c|}{ Several weeldy portious $\mathrm{p}-0.49$} \\
\hline \multirow[t]{6}{*}{ Groups } & \multirow[t]{2}{*}{ Bacall } & nI & 2 & 11 & 27 & 20 & 6 & 66 \\
\hline & & $\%$ & $100.0 \%$ & $50.0 \%$ & $45.8 \%$ & $54.1 \%$ & $66.7 \%$ & $51.2 \%$ \\
\hline & \multirow[t]{2}{*}{ Nures } & $\mathrm{nr}$ & 0 & 11 & 32 & 17 & 3 & 63 \\
\hline & & $\%$ & $0.0 \%$ & $50.0 \%$ & $54.2 \%$ & $45.9 \%$ & $33.3 \%$ & $48.8 \%$ \\
\hline & \multirow[t]{2}{*}{ Total } & $\mathrm{nI}$ & 2 & 22 & 59 & 37 & 9 & 129 \\
\hline & & $\%$ & $100.0 \%$ & $100.0 \%$ & $100.0 \%$ & $100.0 \%$ & $100.0 \%$ & $100.0 \%$ \\
\hline Never $\mathrm{p}$ & & & & & & & & \\
\hline Groups & Bacall & $\mathrm{nr}$ & 2 & 9 & 11 & 4 & 0 & 26 \\
\hline & & $\%$ & $66.7 \%$ & $23.7 \%$ & $25.6 \%$ & $22.2 \%$ & $0.0 \%$ & $23.9 \%$ \\
\hline & Mures & nr & 1 & 29 & 32 & 14 & 7 & 83 \\
\hline & & $\%$ & $33.3 \%$ & $76.3 \%$ & $74.4 \%$ & $77.8 \%$ & $100.0 \%$ & $76.1 \%$ \\
\hline & Total & nf & 3 & 38 & 43 & 18 & & 109 \\
\hline & & $\%$ & $100.0 \%$ & $100.0 \%$ & $100.0 \%$ & $100.0 \%$ & $100.0 \%$ & $100.0 \%$ \\
\hline Total p- & & & & & & & & \\
\hline Groups & Bacaus & $\mathrm{nr}$ & 8 & 58 & 93 & 50 & 22 & 231 \\
\hline & & $\%$ & $80.0 \%$ & $42.0 \%$ & $34.6 \%$ & $39.7 \%$ & $55.0 \%$ & $39.6 \%$ \\
\hline & Mures & $\mathrm{nr}$ & 2 & 80 & 176 & 76 & 18 & 352 \\
\hline & & $\%$ & $20.0 \%$ & $58.0 \%$ & $65.4 \%$ & $60.3 \%$ & $45.0 \%$ & $60.4 \%$ \\
\hline & Total & $\mathrm{nI}$ & 10 & 138 & 269 & 126 & 40 & 583 \\
\hline & & $\%$ & $100.0 \%$ & $100.0 \%$ & $100.0 \%$ & $100.0 \%$ & $100.0 \%$ & $100.0 \%$ \\
\hline
\end{tabular}

\begin{tabular}{|c|c|c|c|c|c|c|c|c|}
\hline \multirow{2}{*}{\multicolumn{3}{|c|}{ Alcohol(vine, beer, liqueur) }} & \multicolumn{5}{|c|}{ BMII } & \multirow[t]{2}{*}{ Total } \\
\hline & & & $<18.5$ & $18.5-24.9$ & $25.0-29.9$ & $30.0-34.9$ & $35.0-39.9$ & \\
\hline \multicolumn{9}{|c|}{ Adaily portion $\mathrm{p}-0.45$} \\
\hline \multirow[t]{6}{*}{ Groups } & \multirow[t]{2}{*}{ Bacalu } & $\mathrm{nr}$ & 1 & 16 & 19 & 17 & 2 & 55 \\
\hline & & $\%$ & $50.0 \%$ & $66.7 \%$ & $61.3 \%$ & $85.0 \%$ & $66.7 \%$ & $68.8 \%$ \\
\hline & \multirow[t]{2}{*}{ Mures } & $\mathrm{nI}$ & 1 & 8 & 12 & 3 & 1 & 25 \\
\hline & & $\%$ & $50.0 \%$ & $33.3 \%$ & $38.7 \%$ & $15.0 \%$ & $33.3 \%$ & $31.2 \%$ \\
\hline & \multirow[t]{2}{*}{ Total } & $\mathrm{nr}$ & 2 & 24 & 31 & 20 & 3 & 80 \\
\hline & & $\%$ & $100.0 \%$ & $100.0 \%$ & $100.0 \%$ & $100.0 \%$ & $100.0 \%$ & $100.0 \%$ \\
\hline \multicolumn{9}{|c|}{ Multiple daily portious $\mathrm{p}-0.77$} \\
\hline \multirow[t]{6}{*}{ Groups } & \multirow[t]{2}{*}{ Bacalu } & nI & 2 & 11 & 18 & 4 & 8 & 43 \\
\hline & & $\%$ & $100.0 \%$ & $91.7 \%$ & $85.7 \%$ & $80.0 \%$ & $100.0 \%$ & $89.6 \%$ \\
\hline & \multirow[t]{2}{*}{ Nures } & $\mathrm{nr}$ & 0 & 1 & 3 & 1 & 0 & 5 \\
\hline & & $\%$ & $0.0 \%$ & $8.3 \%$ & $14.3 \%$ & $20.0 \%$ & $0.0 \%$ & $10.4 \%$ \\
\hline & \multirow[t]{2}{*}{ Total } & $\mathrm{nI}$ & 2 & 12 & 21 & 5 & 8 & 48 \\
\hline & & $\%$ & $100.0 \%$ & $100.0 \%$ & $100.0 \%$ & $100.0 \%$ & $100.0 \%$ & $100.0 \%$ \\
\hline \multicolumn{9}{|c|}{ A weelly portion or less $\mathrm{p}-0.005$} \\
\hline \multirow[t]{6}{*}{ Groups } & \multirow[t]{2}{*}{ Bacaus } & nI & 1 & 11 & 18 & 5 & 6 & 41 \\
\hline & & $\%$ & $100.0 \%$ & $26.2 \%$ & $15.7 \%$ & $10.9 \%$ & $46.2 \%$ & $18.9 \%$ \\
\hline & \multirow[t]{2}{*}{ Mures } & $\mathrm{nr}$ & 0 & 31 & 97 & 41 & 7 & 176 \\
\hline & & $\%$ & $0.0 \%$ & $73.8 \%$ & $84.3 \%$ & $89.1 \%$ & $53.8 \%$ & $81.1 \%$ \\
\hline & \multirow[t]{2}{*}{ Total } & $\mathrm{nr}$ & 1 & 42 & 115 & 46 & 13 & 217 \\
\hline & & $\%$ & $100.0 \%$ & $100.0 \%$ & $100.0 \%$ & $100.0 \%$ & $100.0 \%$ & $100.0 \%$ \\
\hline \multicolumn{9}{|c|}{ Several weeldy portious p-0.49 } \\
\hline \multirow[t]{6}{*}{ Groups } & \multirow[t]{2}{*}{ Bacau } & nI & 2 & 11 & 27 & 20 & 6 & 66 \\
\hline & & $\%$ & $100.0 \%$ & $50.0 \%$ & $45.8 \%$ & $54.1 \%$ & $66.7 \%$ & $51.2 \%$ \\
\hline & \multirow[t]{2}{*}{ Nures } & $\mathrm{ar}$ & 0 & 11 & 32 & 17 & 3 & 63 \\
\hline & & $\%$ & $0.0 \%$ & $50.0 \%$ & $54.2 \%$ & $45.9 \%$ & $33.3 \%$ & $48.8 \%$ \\
\hline & \multirow[t]{2}{*}{ Total } & $\mathrm{nr}$ & 2 & 22 & 59 & 37 & 9 & 129 \\
\hline & & $\%$ & $100.0 \%$ & $100.0 \%$ & $100.0 \%$ & $100.0 \%$ & $100.0 \%$ & $100.0 \%$ \\
\hline Never $\mathrm{p}$ & & & & & & & & \\
\hline Groups & Bacall & $\mathrm{nI}$ & 2 & 9 & 11 & 4 & 0 & 26 \\
\hline & & $\%$ & $66.7 \%$ & $23.7 \%$ & $25.6 \%$ & $22.2 \%$ & $0.0 \%$ & $23.9 \%$ \\
\hline & Mures & $\mathrm{nr}$ & 1 & 29 & 32 & 14 & 7 & 83 \\
\hline & & $\%$ & $33.3 \%$ & $76.3 \%$ & $74.4 \%$ & $77.8 \%$ & $100.0 \%$ & $76.1 \%$ \\
\hline & Total & $\mathrm{nr}$ & 3 & 38 & 43 & 18 & & 109 \\
\hline & & $\%$ & $100.0 \%$ & $100.0 \%$ & $100.0 \%$ & $100.0 \%$ & $100.0 \%$ & $100.0 \%$ \\
\hline Total p- & & & & & & & & \\
\hline Groups & Bacalu & $\mathrm{nr}$ & 8 & 58 & 93 & 50 & 22 & 231 \\
\hline & & $\%$ & $80.0 \%$ & $42.0 \%$ & $34.6 \%$ & $39.7 \%$ & $55.0 \%$ & $39.6 \%$ \\
\hline & Mures & $\mathrm{nI}$ & 2 & 80 & 176 & 76 & 18 & 352 \\
\hline & & $\%$ & $20.0 \%$ & $58.0 \%$ & $65.4 \%$ & $60.3 \%$ & $45.0 \%$ & $60.4 \%$ \\
\hline & Total & $\mathrm{nr}$ & 10 & 138 & 269 & 126 & 40 & 583 \\
\hline & & $\%$ & $100.0 \%$ & $100.0 \%$ & $100.0 \%$ & $100.0 \%$ & $100.0 \%$ & $100.0 \%$ \\
\hline
\end{tabular}

Table 4

COMPARATIVE TABLE OF PHYSICAL ACTIVITY FREQUENCY OF THE PARTICIPANTS GROUPED BY THEIR BMI VALUES
Table 5 
Regarding alcohol consumption, not quite significant relationship could be revealed for both areas. For the population in Bacau p- 0.065 and for those living in Mures p0.077 .

We identified significant difference regarding alcohol consumption once or several times per week (p-0.005), the frequency being higher in the subjects living in Tirgu Mures and surroundings. In case of other options, no significant differences could be observed, but daily consumption was higher in the population from Bacau and surroundings (table 5 ).

The novelty of this study makes the authors believe it is a first-time possibility to assess the risk factors of the rapidly changing lifestyle of the modern society in two distinct regions of Romania. It is a notable fact, that the life quality is slightly different in the two regions, Tirgu Mures being the region with the higher quality of life, and it is also a university centre, expectedly providing better health care support and higher quality of health care services.

The experimental side of our study consists in assessing the lifestyle habits, socio-economical factors and state of health of the included subjects using validated, complex questionnaire. It is among the first studies in Romania with such complexity and size, that aims the analysis of the previously presented aspects of general population, and to conclude by recommending solutions in order to prevent the incorrect lifestyle habits induced diseases that affect the entire society.

According to recent research data, several unhealthy eating habits, like irregular meals or watching television during meals, are very common in the Romanian population $[15,16]$.

Other studies found association between eating breakfast and other healthy lifestyle habits like being a nonsmoker, regular exerciser, and trying to control weight [17]. In our study this association may be present, but our questionnaire did not permit to test this kind of associations. A recently published article evidenced the relationship between skipping breakfast and increasing body mass index and waist circumference, breakfast skippers were more likely to gain weight in the following 5 years than non-skippers [18].

Not only the frequency, but also the type of the snack is crucial from the point of view of weight gain. A recent study focused on the relationship between snack-food choices and BMI. The results showed that participants having BMI over $25 \mathrm{~kg} / \mathrm{m}^{2}$ consumed higher amounts of crisps, sweets, ice-cream, chocolate and lower amounts of nuts and yoghurt compared to the group of normalweight subjects [19].

\section{Conclusions}

More than 2/3 of the studied population is overweight or obese, which is a clear indicator of the negative influence of the modern lifestyle habits on the state of health. The physical activity of the questioned subjects is quite acceptable, due to the high rate of daily regular exercise present in our study group, however the BMI values are disproportionately high, leading to a conclusion that even though the beneficial effects of physical activity, other factors are also strongly influencing the general state of health of the individuals.

Daily and weeklyalcohol consumption is frequent in our study group. A high percentage of the subjects consume their breakfast daily, and snack consumption between meals had a much lower ratio.

Comparing the results of the two distinct geographical regions we can state that: regarding breakfast consumption those individuals who have higher BMI values responded "sometimes" more frequently compared to Bacau, and regarding the snack consumption between main meals, the subjects with low BMI values $(<18.5 \mathrm{~kg} /$ $\mathrm{m}^{2}$ ) of Bacau presented more frequently a rare or lack consumption of snacks between meals, compared to Tirgu Mures and surroundings. Physical activity is similar regarding its frequency in the two geographical areas, differences being present in only one case: in Tirgu Mures and surroundings low BMI subjects have higher rates of rarely or no physical activity at all than in Bacau. There are no significant differences regarding alcohol consumption in the two regions, except one case: in Tirgu Mures higher cases of subjects consume alcohol once or several times per week than in Bacau.

Daily and weekly several times alcohol consumption is quite frequent in both, Transylvanian and Moldavian study group, and an action of intervention is required in order to reduce its frequency. High combined rates of lacking breakfastneeds to be reduced for the correction of dietary habits of both study groups, and to reduce the impact of this unhealthy behavior.

The high frequency of daily physical activity of respondents is a good sign that local and also global instructional and educational interventions targeting the development of healthier lifestyle habits in general population are worhty and needed to reduce the risk factors associated with the development of cardiovascular and metabolic diseases and reducing the burden upon the society of these diseases.

\section{References}

1.TILINCA, M.C., VOIDAZAN, S., NYULAS, K., TILINCA R.M., NEMESNAGY ENIKO, Study on metabolic parameters in obese patients compared to normal weight subjects before and after joint replacement surgery, Rev. Chim. (Bucharest), 69, no 4, 2018, p. 944 947

2.PIKO, B., Vedofaktorok nyomaban. A karos szenvedelyek es egeszsegfejlesztes serdulokorban. Ed. L'Harmattan, Budapesta, 2010 3.MOONEY, L.A., KNOX, D., SCHACHT, C., Understanding Social Problems, Seventh Edition, Wadsworth, Cengage Learning, Belmont, 2011

4.FOGARASI, E., CROITORU, M.D., FULOP, I., NEMES-NAGY E., TRIPON R.G., SIMON-SZABO, Z., MUNTEAN D.L., Malondialdehyde levels can be measured in serum and saliva by using a fast HPLC method with visible detection, Rev. Rom. Med. Lab., 24, nr. 3, 2016, p. 319-326

5.OGDEN, J., Health Psychology, Open University Pres, McGraw-Hill, 2007

6.BANDURA, A., The growing centrality of self-regulation in Health Promotion and disease prevention. The European Health Psychology, 9, I, 2005

7.MATARAZZO, J.D., WEISS, S.M., HERD, J .A., et al., Behavioral health: $A$ handbook of health enhancement and disease prevention. New York, Wiley, 1984

8.TARCEA, M., FAZAKAS, Z., SZUCS, V., KOVACS, Z., NEMES-NAGY, E., OLAH, P., TILINCA, M., GUINE, R., Mean dietary fiber intake of romanian adults - results of a survey questionnaire, Rev. Chim. (Bucharest), 68, no. 9, 2017, p. 2083-2087

9.MACKOWIAK, K., TORLINSKA-WALKOWIAK, N., TORLINSKA, B., Dietary fibre as an important constituent of the diet. Adv. Hyg. Exp. Med., 70, 2016, p. 104-109

10.SAMASCA, G., SUR, G., LUPAN, I., TILINCA, M., DELEANU, D., Celiac disease as an autoimmune condition. Cent. Eur. J. Immunol., 39, nr. 3, 2014, p. 396-399

11.FELT, U., FEIDER, K., PENKLER, M., How differences matter: tracing diversity practices in obesity treatment and health promotion, Sociol. Health Iln., 39, nr. 1, 2017, p. 127-142 
12.WEE C.C., DAVISR.B., CHIODI S., et al., Sex, race, and the adverse effects of social stigma vs. other quality of life factors among primary care patients with moderate to severe obesity, J. Gen. Int. Med., 30, nr. 2, 2015, p. 229-35

13.NEMES-NAGY, E., FAZAKAS, Z, BALOGH-SAMARGHITAN, V., HIGGINS, T., Comparison of four chromatographic methods used for measurement of glycated hemoglobin. Rev. Rom. Med. Lab., 24, nr. 4, 2016, p. 431-9.

14.BALINT, I., NEMES-NAGY, E., CSIBI, M., TUBAK, N., OLAH, P., PREG, Z., GERMAN SALO, M., TILINCA, M., ORMENISAN, A., ABRAM, Z., FAZAKAS, Z., The Role of Diet and Lifestyle Habits in Prevention of Cardiovascular Diseases in Moldavian and Transylvanian Population, Rev. Chim. (Bucharest), 68, no. 7, 2017, p. 1460-1465

15.ROMAN, G., BALA, C., CRETEANU, G., ACHIMAS CADARIU, A., Obesity and Health-Related Lifestyle Factors in the General Population in Romania: a Cross Sectional Study, Acta Endo (Buc), 11, nr. 1, 2015, p. $64-72$
16.TILINCA, M.C., PAL, S., PREG, Z., NEMES-NAGY, E., The Relationship of Metabolic and Endocrine Parameters with Associated Diseases in Diabetes Mellitus, Rev. Chim. (Bucharest), 69, no. 5, 2018, p. 1288-91 17.SONG, W.O., CHUN, O.K., OBAYASHI, S., et al., Is Consumption of Breakfast Associated with Body Mass Index in US Adults?, J. Am. Diet Assoc., 105, nr. 9, 2005, p. 1373-82

18.SAKURAI, M., YOSHITA, K., NAKAMURA, K., etal., Skipping breakfast and 5-year changes in body mass index and waist circumference in J apanese men and women: Skipping breakfastand body weight gain., Obes. Sci. Pract., 3, nr. 2, 2017, p. 162-70

19.0'CONNOR, L., BRAGE, S., GRIFFIN, S.J ., et al., The cross-sectional association between snacking behaviour and measures of adiposity: the Fenland Study, UK, British J. Nutrition. 114, nr. 8, 2015, p. 1286-93

Manuscript received: 15.03 .2018 